\title{
TABLES FOR THE CALCULATION OF THE PROBABILITY TO BE USED IN THE MODIFIED BONFERRONI'S TEST
}

\author{
Armando Conagin ${ }^{1}$
}

\section{ABSTRACT}

The present paper presents 8 tables to furnish $\mathrm{P}(\mathrm{F})$ values to be utilized in the calculation of BM and BMP to be used in comparison of means. Beside those we present Table 1 to show the performance of the BM and BMP tests proposed by Conagin $(1998,1999)$ as an improvement of Bonferroni's test.

\section{RESUMO}

O presente artigo apresenta 8 Tabelas (2 a 9) com vistas a fornecer o valor $\mathrm{P}(\mathrm{F})$ a ser utilizado em cálculo dos testes $\mathrm{B}_{\mathrm{M}}$ e $\mathrm{B}_{\mathrm{MP}}$.

Apresenta também a tabela 1 com resultados que permitem sejam comparados os poderes discriminativos desses dois testes com os ter cos LSD, Duncan, SNK, Tukey, Bonferroni e Dunnett.

Palavras-chave: poder discriminativo de testes estatísticos, cálculo de BM e BMP, valores de $\mathrm{P}(\mathrm{F})$ em 8 Tabelas para utilização nesses cálculos.

\section{INTRODUCTION}

Conagin (1998 and 1999) has proposed a modification of Bonferroni's test to be used in the comparison among treatment means in

1. Agronomist and Applied Statistician, retired, Instituto Agronômico, Campinas, SP, Brazil. 
experiments. The method was described in two articles published in $\mathbf{R e}$ vista de Agricultura (Conagin, 1998 and 1999). He proved that his test has a higher discriminative power than other usual tests, as shows Table 1. But the calculation of the probability level to be used required the use of PROBF FUNCTION of the SAS Program (SAS, 1990).

Now he presents tables ( 2 to 9 ) to make easier the application of his test.

\section{Example}

Let us assume an experiment with $\mathrm{t}=6$ treatments in 3 randomized blocks, with observed $\mathrm{F}_{\mathrm{o}}=9.72$, and 10 degrees of freedom (df) for the Residual. Assume also that $\mathrm{k}=4$ contrasts of 2 means are to be tested. The level of probability for the usual Bonferroni's test would be $\alpha$ ' $=\alpha / \mathrm{K}$ $=0,05 / 4=0,0125$. But in Conagin's test this level is changed to

$$
\delta_{B M}=\frac{\alpha}{K}[1+P(F)]=\frac{0,05}{4}[1+P(F)]
$$

The values of $P(F)$ are given for different values of $t$ (number of treatments), of $r$ (number of replications) and $F_{0} / F_{c}=$ ratio of $F$ obtained $\mathrm{F}_{\mathrm{o}}$ and table value $\mathrm{F}_{\mathrm{c}}$ at $\alpha=5 \%$ level of probability.

In the example shown we have $\mathrm{t}=6$ treatments, $\mathrm{r}=3$ replications, $\mathrm{F}_{\mathrm{o}} / \mathrm{F}_{\mathrm{c}}=9.72 / 3.33=2.92$, where $\mathrm{F}_{\mathrm{c}}$ refers to $5 \%$ probability, with $5 \mathrm{df}$ for treatments, and $10 \mathrm{df}$ for error.

Table 3 , with $t=6$ treatments, $r=3$ replications, $F_{o} / F_{c}=2,92$ gives:

$$
\begin{aligned}
& \frac{\mathrm{F}_{\mathrm{o}}}{\mathrm{F}_{\mathrm{c}}}=2.5 \rightarrow P(F)=0.470 \\
& \frac{\mathrm{F}_{\mathrm{o}}}{\mathrm{F}_{\mathrm{c}}}=3.0 \rightarrow P(F)=0.478
\end{aligned}
$$


Linear interpolation gives $\mathrm{P}(\mathrm{F})=0.477$.

So, we obtain:

$$
\begin{aligned}
\delta_{\mathrm{BM}} & =\alpha[1+\mathrm{P}(\mathrm{F})] / 4 \\
& =0.05(1+0.477) / 4 \\
& =0.0185
\end{aligned}
$$

Now the usual t-test table gives for $\mathrm{df}=10$ :

$$
\begin{aligned}
& \alpha=1 \% \rightarrow \mathrm{t}=3.17 \\
& \alpha=2 \% \rightarrow \mathrm{t}=2.76
\end{aligned}
$$

Linear interpolation gives $\mathrm{t}=2.82$. Thus the $1 \mathrm{sd}$ for the test would be:

$$
l s d=\frac{2.82 \times s}{\sqrt{3}}=1.63 s \quad
$$

where $\mathbf{s}$ is the standard deviation. The usual Bonferroni's test would give $1 \mathrm{sd}=1.76 \mathrm{~s}$, a higher value. 
Table 1. Result obtained when $\mathrm{CV}=10 \%$, for $\mathrm{r}=3$, $\mathrm{df}=34(\mathrm{t}=18)$, and $r=6$ and $d f=68(t=18)$ when $K=4$, of $40 \%, 30 \%$, $20 \%, 10 \%$ of the respective treatment mean and the control mean. Values in the cells represent the discriminative power in percentage in 200 simulated experiments whith $r=3$ and 100 experiments with $r=6$.

\begin{tabular}{|c|c|c|c|c|c|c|c|c|}
\hline & \multicolumn{3}{|c|}{$r=3, \mathrm{df}=34$} & \multicolumn{3}{c|}{$r=6, \mathrm{df}=68$} \\
\hline Dif & $40 \%$ & $30 \%$ & $20 \%$ & $10 \%$ & $40 \%$ & $30 \%$ & $20 \%$ & $10 \%$ \\
\hline LSD & 100 & 96 & 70 & 23.5 & 100 & 99 & 95 & 40 \\
Duncan & 99.5 & 95 & 59 & 13 & 100 & 99 & 90 & 25 \\
SNK & 95 & 65.5 & 19.5 & 2 & 100 & 99 & 68 & 8 \\
Tukey & 89.5 & 53 & 13.5 & 1.5 & 100 & 96 & 50 & 6 \\
Bonferroni & 82.5 & 45.5 & 10.5 & 1 & 100 & 95 & 41 & 4 \\
Dunnett & 97 & 74 & 33.5 & 4.5 & 100 & 99 & 76 & 13 \\
BM & 99.5 & 90 & 56.5 & 7.5 & 100 & 99 & 87 & 26 \\
BMP & 98.5 & 88 & 55.5 & 11 & 100 & 99 & 88 & 25 \\
\hline
\end{tabular}

$r=$ number of replications;

$\mathrm{t}=$ number of treatments;

$\mathrm{df}=$ nunber of degrees of freedom;

LSD = least significant difference by t-test;

SNK = Student, Newman, Keuls test;

$\mathrm{BM}=$ Modified Bonferroni's test;

$\mathrm{BMP}=$ partial modified Bonferroni's test. 


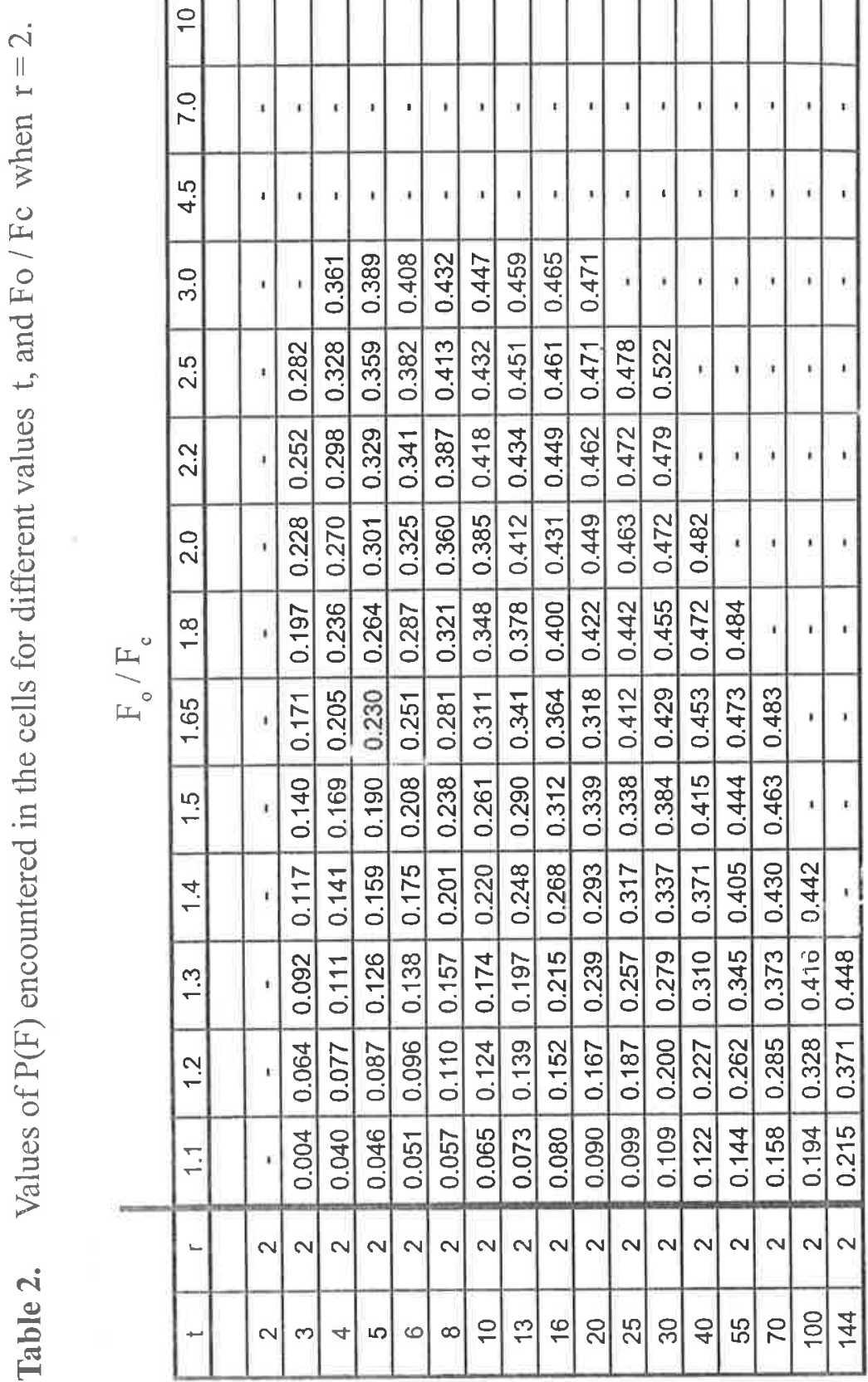




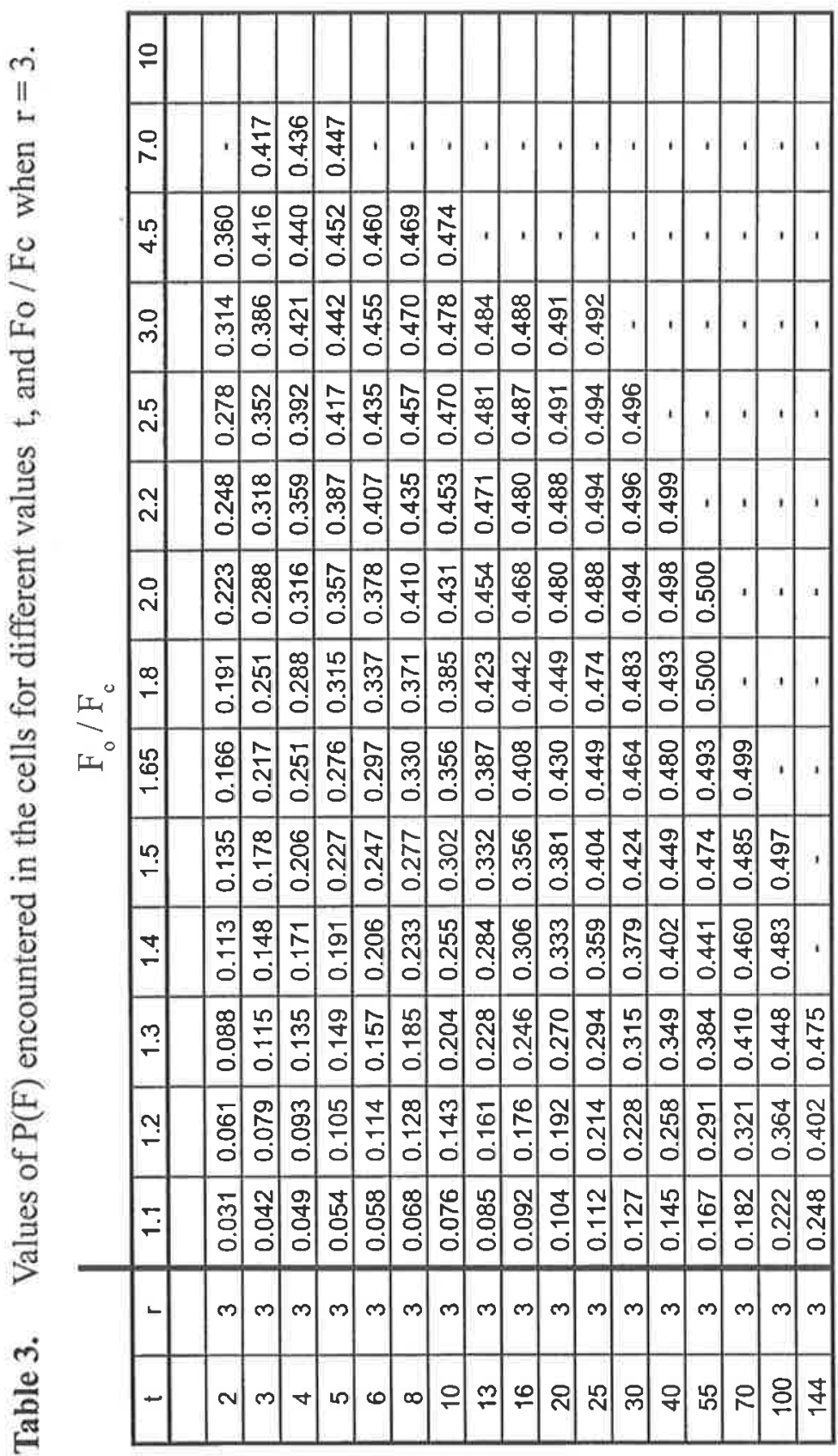




\begin{tabular}{|c|c|c|c|c|c|c|c|c|c|c|c|c|c|c|c|c|c|c|}
\hline$\therefore$ & 으 & $\begin{array}{l}8 \\
\text { ఫे } \\
\end{array}$ & 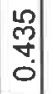 & . & 1 & 1 & . & 1 & ' & . & . & . & t & 1 & I & ' & I & . \\
\hline 5 & 0 & 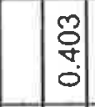 & \begin{tabular}{l}
0 \\
\multirow{y}{*}{} \\
0
\end{tabular} & $\begin{array}{l}10 \\
8 \\
0 \\
0\end{array}$ & $\begin{array}{l}8 \\
0 \\
0 \\
0\end{array}$ & $\begin{array}{l}9 \\
0 \\
0 \\
0\end{array}$ & $\cdot$ & 1 & 1 & 1 & , & . & 1 & . & 1 & . & ' & . \\
\hline 0 & $\mid \begin{array}{l}n \\
\dot{q}\end{array}$ & $\begin{array}{l}\text { 今े } \\
\text { ले } \\
\text { ○े }\end{array}$ & 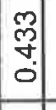 & $\begin{array}{l}\overline{0} \\
\dot{0} \\
0\end{array}$ & $\begin{array}{l}0 \\
\frac{8}{8} \\
0\end{array}$ & \begin{tabular}{l}
$\infty$ \\
\multirow{y}{0}{} \\
0
\end{tabular} & 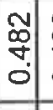 & 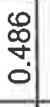 & . & . & . & . & 1 & ' & . & 1 & . & . \\
\hline 0 & $\begin{array}{c}0 \\
\text { ले }\end{array}$ & $\begin{array}{l}\text { फ్ } \\
\text { రे }\end{array}$ & $\begin{array}{l}\infty \\
\\
0 \\
0\end{array}$ & $\begin{array}{l}\infty \\
+ \\
0\end{array}$ & \begin{tabular}{l}
\multirow{0}{0}{} \\
$\dot{0}$ \\
0
\end{tabular} & \begin{tabular}{l} 
\pm \\
\multirow{0}{*}{} \\
0
\end{tabular} & 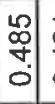 & $\begin{array}{l}\check{g} \\
\dot{0}\end{array}$ & \begin{tabular}{l}
5 \\
\multirow{8}{0}{} \\
0
\end{tabular} & 守 & $\begin{array}{l}\text { g } \\
\text { ஓे. } \\
\text { ○ }\end{array}$ & , & ' & ' & . & ' & . & . \\
\hline كُس & $\mid \begin{array}{l}10 \\
\sim \\
\sim\end{array}$ & $\frac{n}{m}$ & $\begin{array}{c}\mathbb{N} \\
\tilde{N} \\
0 \\
0\end{array}$ & $\frac{\sigma}{8}$ & $\begin{array}{l}\tilde{y} \\
\dot{0}\end{array}$ & $\begin{array}{l}0 \\
5 \\
0 \\
0\end{array}$ & \begin{tabular}{l}
$\frac{1}{\Omega}$ \\
\multirow{8}{*}{} \\
0
\end{tabular} & $\begin{array}{l}\infty \\
0 \\
\dot{0}\end{array}$ & 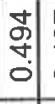 & $\begin{array}{l}\text { Oे } \\
\text { ○े }\end{array}$ & $\begin{array}{l}8 \\
\text { 유 } \\
0\end{array}$ & \begin{tabular}{l|l} 
गे \\
గొ \\
0
\end{tabular} & 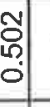 & , & . & 1 & ' & . \\
\hline$\frac{\pi}{3}$ & va & 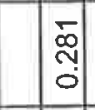 & 守 & $\begin{array}{c}n \\
0 \\
\tilde{m} \\
0 \\
0\end{array} \mid$ & $\frac{-}{8}$ & \begin{tabular}{l} 
요 \\
\multirow{0}{0}{} \\
\end{tabular} & 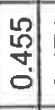 & $\begin{array}{l}\bar{T} \\
\dot{0} \\
0\end{array}$ & $\begin{array}{l}0 \\
0 \\
\vdots \\
0\end{array}$ & $\begin{array}{l}m \\
\stackrel{9}{q} \\
0\end{array}$ & $\begin{array}{l}\text { o } \\
\text { 寸े. } \\
0\end{array}$ & 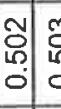 & 勇 & 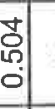 & . & I & , & . \\
\hline$\frac{5}{50}$ & 어 & ָָ ָָ & गे & $\begin{array}{l}\text { m్ల } \\
\text { లై } \\
0\end{array}$ & $\begin{array}{l}\Omega \\
\tilde{m} \\
0\end{array}$ & $\begin{array}{l}8 \\
\text { 巳̣ } \\
0\end{array}$ & $\begin{array}{c}\tilde{m} \\
\dot{0}\end{array}$ & $\begin{array}{l}0 \\
\text { ○ी } \\
0 \\
0\end{array}$ & \begin{tabular}{l}
0 \\
\multirow{8}{*}{} \\
0
\end{tabular} & 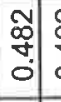 & $\begin{array}{l}\text { कै } \\
\text { จे }\end{array}$ & 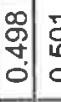 & பீ & 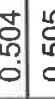 & $\begin{array}{l}10 \\
0 \\
0 \\
0\end{array}$ & . & , & 1 \\
\hline$I^{\circ}$ & $\stackrel{\infty}{-}$ & స̃. & $\begin{array}{c}\text { m. } \\
\text { ஸ̦ } \\
0\end{array}$ & $\begin{array}{l}\text { ஓ్ } \\
\text { లె } \\
0\end{array}$ & लె & $\begin{array}{l}\infty \\
\text { ஸ̂ } \\
0 \\
0\end{array}$ & $\begin{array}{c}\bar{\rho} \\
\text { ஸे }\end{array}$ & $\begin{array}{l}\frac{10}{7} \\
0 \\
0\end{array}$ & $\begin{array}{l}\check{q} \\
\dot{0}\end{array}$ & \begin{tabular}{l}
$\infty$ \\
$\stackrel{0}{0}$ \\
\multirow{0}{*}{}
\end{tabular} & 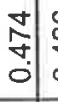 & 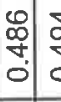 & ४ & రీ & $\begin{array}{l}\text { ఫे } \\
\text { ధ. } \\
0\end{array}$ & $\begin{array}{l}6 \\
0 \\
6 \\
0\end{array}$ & . & , \\
\hline$\Psi^{\circ}$ & $\mid$\begin{tabular}{|l|} 
\\
\hdashline \\
\hdashline \\
-
\end{tabular} & $\begin{array}{l}\mathscr{E} \\
\frac{0}{0} \\
\end{array}$ & 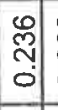 & $\begin{array}{l}\stackrel{g}{ } \\
\text { ஸ̣ } \\
0\end{array}$ & $\begin{array}{l}\stackrel{\sharp}{~} \\
\vdots \\
0\end{array}$ & mo & $\begin{array}{l}0 \\
\text { ల్ } \\
\text { లె| }\end{array}$ & $\begin{array}{l}\mathbb{N} \\
\text { m } \\
0\end{array}$ & $\begin{array}{c}+ \\
\stackrel{+}{+} \\
\dot{0}\end{array}$ & 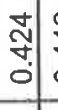 & $\begin{array}{l}0 \\
\dot{y} \\
0\end{array}$ & \begin{tabular}{l}
9 \\
0 \\
\hdashline \\
0
\end{tabular} & \begin{tabular}{l}
$\mathscr{0}$ \\
\multirow{5}{*}{} \\
0
\end{tabular} & 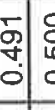 & $\begin{array}{l}8 \\
8 \\
0 \\
0\end{array}$ & $\begin{array}{l}\text { m } \\
0 \\
0 \\
0\end{array}$ & , & . \\
\hline$\Xi$ & مُمـا & $\frac{\overline{5}}{0}$ & $\frac{m}{\sigma}$ & 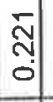 & 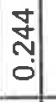 & 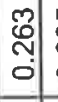 & 只 & $\begin{array}{l}\infty \\
\stackrel{\infty}{0} \\
0\end{array}$ & $\begin{array}{l}\infty \\
\text { mे } \\
\stackrel{0}{0}\end{array}$ & $\begin{array}{l}\mathrm{N} \\
\mathrm{m} \\
\mathrm{0}\end{array}$ & $\begin{array}{c}\text { హे } \\
\text { o. }\end{array}$ & $\begin{array}{c}\bar{N} \\
\text { vे } \\
0\end{array}$ & $\begin{array}{c}\infty \\
\stackrel{\infty}{m} \\
0 \\
0\end{array}$ & \begin{tabular}{l|l}
2 & \\
0 \\
0 \\
0
\end{tabular} & 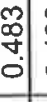 & $\begin{array}{l}m \\
\text { के } \\
0 \\
0\end{array}$ & $\begin{array}{l}m \\
0 \\
1 \\
0 \\
0\end{array}$ & . \\
\hline 岕 & $\stackrel{\nabla}{-}$ & $\stackrel{\leftarrow}{\stackrel{\leftarrow}{0}}$ & \begin{tabular}{|l|}
0 \\
0 \\
0 \\
\end{tabular} & $\frac{m}{\infty}$ & $\begin{array}{l}m \\
\stackrel{9}{0} \\
0\end{array}$ & స్ & $\begin{array}{l}0 \\
\stackrel{0}{0} \\
0\end{array}$ & $\begin{array}{c}\pi \\
0\end{array}$ & $\begin{array}{c}- \\
\text { లె }\end{array}$ & $\begin{array}{l}\stackrel{m}{m} \\
\dot{0}\end{array}$ & \begin{tabular}{l}
$\infty$ \\
\multirow{j}{*}{} \\
0
\end{tabular} & ले & 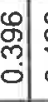 & 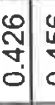 & $\begin{array}{l}\infty \\
0 \\
0 \\
0\end{array}$ & $\begin{array}{l}N \\
\dot{N} \\
\dot{0}\end{array}$ & $\begin{array}{l}\overleftarrow{\sigma} \\
\dot{0} \\
0\end{array}$ &, \\
\hline$\frac{\tilde{d}}{\mathbb{0}}$ & $\stackrel{m}{\circ}$ & $\begin{array}{l}\infty \\
\stackrel{9}{0} \\
0 \\
0\end{array}$ & 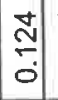 & $\frac{7}{0}$ & $\stackrel{\circ}{\stackrel{\circ}{\circ}}$ & $\begin{array}{l}\Omega \\
\stackrel{\Omega}{\rho}\end{array}$ & $\frac{\hat{\sigma}}{0}$ & స̃. & ণ্ণ & 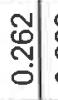 & 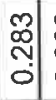 & $\begin{array}{l}\text { ஓ् } \\
\text { ○े }\end{array}$ & $\begin{array}{l}m \\
m \\
m \\
0\end{array}$ & $\begin{array}{c}1 \\
0 \\
0\end{array}$ & $\begin{array}{l}8 \\
\text { ఫे } \\
0\end{array}$ & 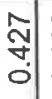 & $\begin{array}{l}8 \\
\stackrel{0}{+} \\
0\end{array}$ & 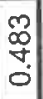 \\
\hline$\frac{1}{0}$ & $\stackrel{N}{\top}$ & $\begin{array}{l}0 \\
\$ \\
0 \\
0 \\
0\end{array}$ & $\begin{array}{l}0 \\
0 \\
0 \\
0 \\
0\end{array}$ & $\begin{array}{l}\text { D } \\
\text { ○ } \\
0\end{array}$ & $\frac{0}{0}$ & $\begin{array}{l}\text { ㅇ } \\
\vdots \\
0\end{array}$ & $\stackrel{\hat{m}}{0}$ & $\begin{array}{l}\overline{0} \\
0 \\
0\end{array}$ & $\stackrel{N}{N}$ & $\begin{array}{l}\mathscr{D} \\
\vdots \\
0\end{array}$ & $\begin{array}{l}\infty \\
\text { ஸุ. } \\
0\end{array}$ & \begin{tabular}{c}
\multirow{N}{N}{} \\
N̦ \\
0
\end{tabular} & 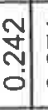 & స̃. & Г̄ & $\begin{array}{l}\text { D్ } \\
\text { లె } \\
\text { ○े }\end{array}$ & $\begin{array}{l}n \\
n \\
m \\
0 \\
0\end{array}$ & $\begin{array}{l}\frac{\sigma}{8} \\
\dot{8} \\
0\end{array}$ \\
\hline$\stackrel{2}{\rightleftarrows}$ & 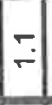 & $\begin{array}{l}10 \\
\text { مొ } \\
0\end{array}$ & 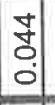 & $\begin{array}{l}N \\
\text { ஸ્. } \\
0 \\
0\end{array}$ & $\begin{array}{l}\infty \\
0 \\
0 \\
0 \\
0\end{array}$ & $\begin{array}{l}\mathbb{N} \\
0 \\
0\end{array}$ & $\begin{array}{l}N \\
0 \\
0\end{array}$ & $\begin{array}{l}\bar{\infty} \\
0 \\
0\end{array}$ & $\begin{array}{l}\infty \\
0 \\
0 \\
0 \\
0\end{array}$ & $\begin{array}{l}\infty \\
\text { o. } \\
0 \\
0\end{array}$ & $\stackrel{0}{\circ}$ & 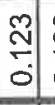 & $\begin{array}{c}0 \\
\\
\\
0\end{array}$ & $\begin{array}{l}9 \\
\\
\end{array}$ & $\begin{array}{l}\Phi \\
\square \\
\end{array}$ & $\begin{array}{l}m \\
\stackrel{\rho}{\circ} \\
0\end{array}$ & $\begin{array}{l}\text { ָ̃ } \\
\text { ஸ̃ }\end{array}$ & $\begin{array}{l}\text { ه } \\
\stackrel{0}{0} \\
0 \\
\end{array}$ \\
\hline & ᄂ & 寸 & ष & 寸 & $\nabla$ & $\nabla$ & ষ & $\nabla$ & $\nabla$ & $\nabla$ & $\forall$ & $\nabla$ & $\nabla$ & 寸 & $\nabla$ & $\nabla$ & $\nabla$ & $\nabla$ \\
\hline 政 & -1 & $\sim$ & $m$ & $\checkmark$ & (n) & $\infty$ & $\infty$ & 임 & $\stackrel{m}{\rightleftharpoons}$ & 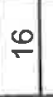 & 尺̊ & $\stackrel{10}{\sim}$ & లి & 웜 & ก & P & 잉 & \& \\
\hline
\end{tabular}




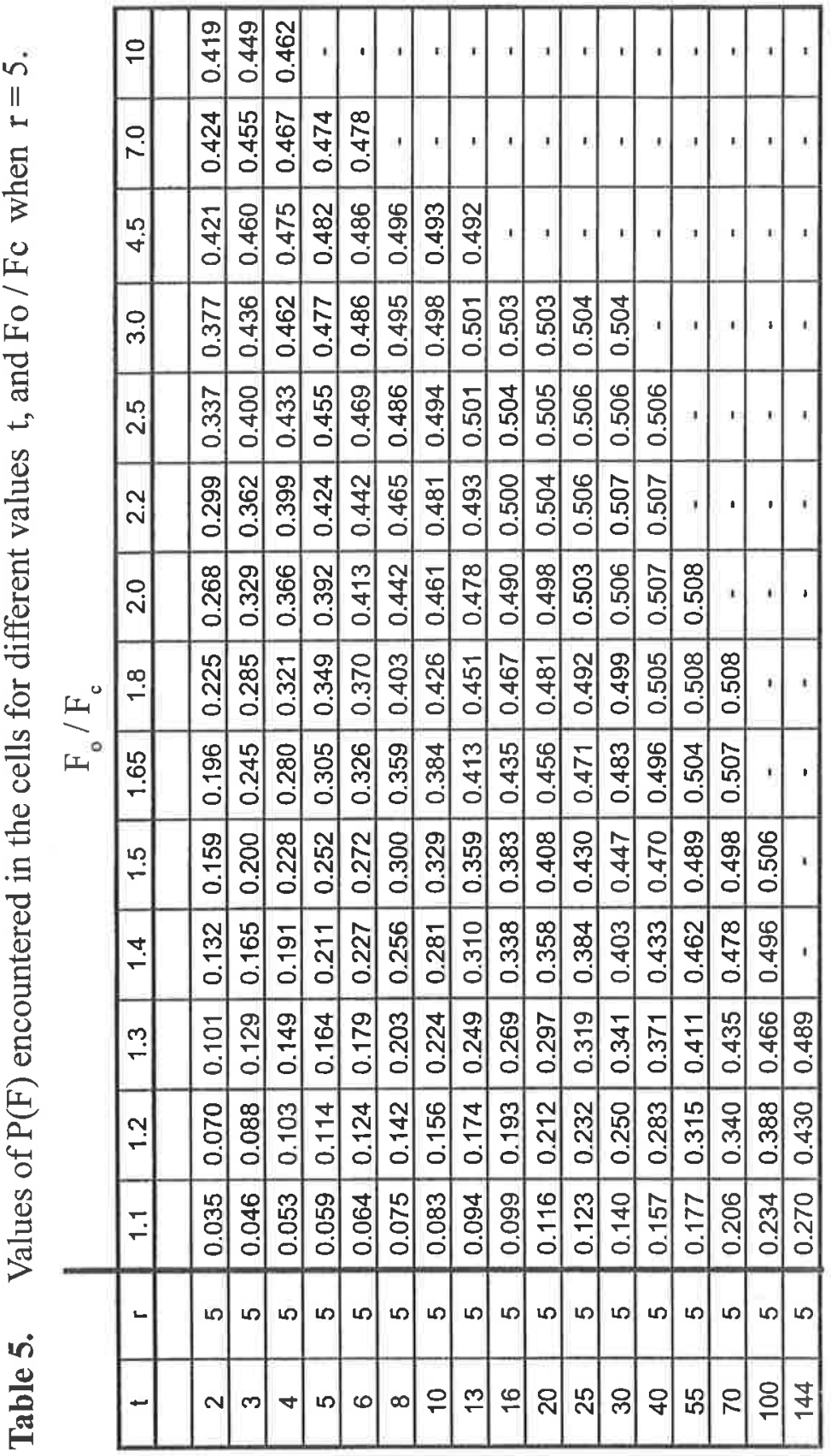




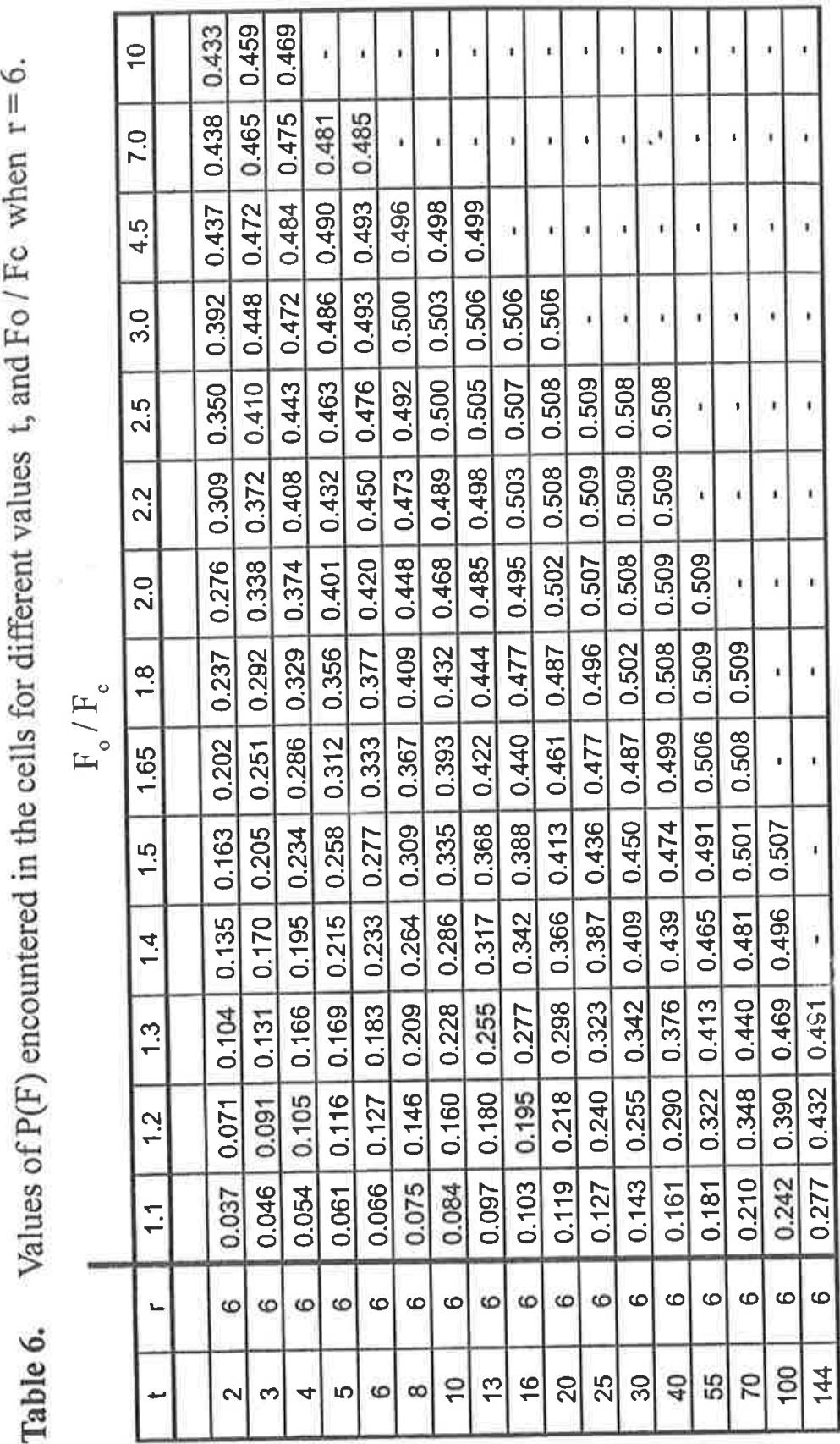




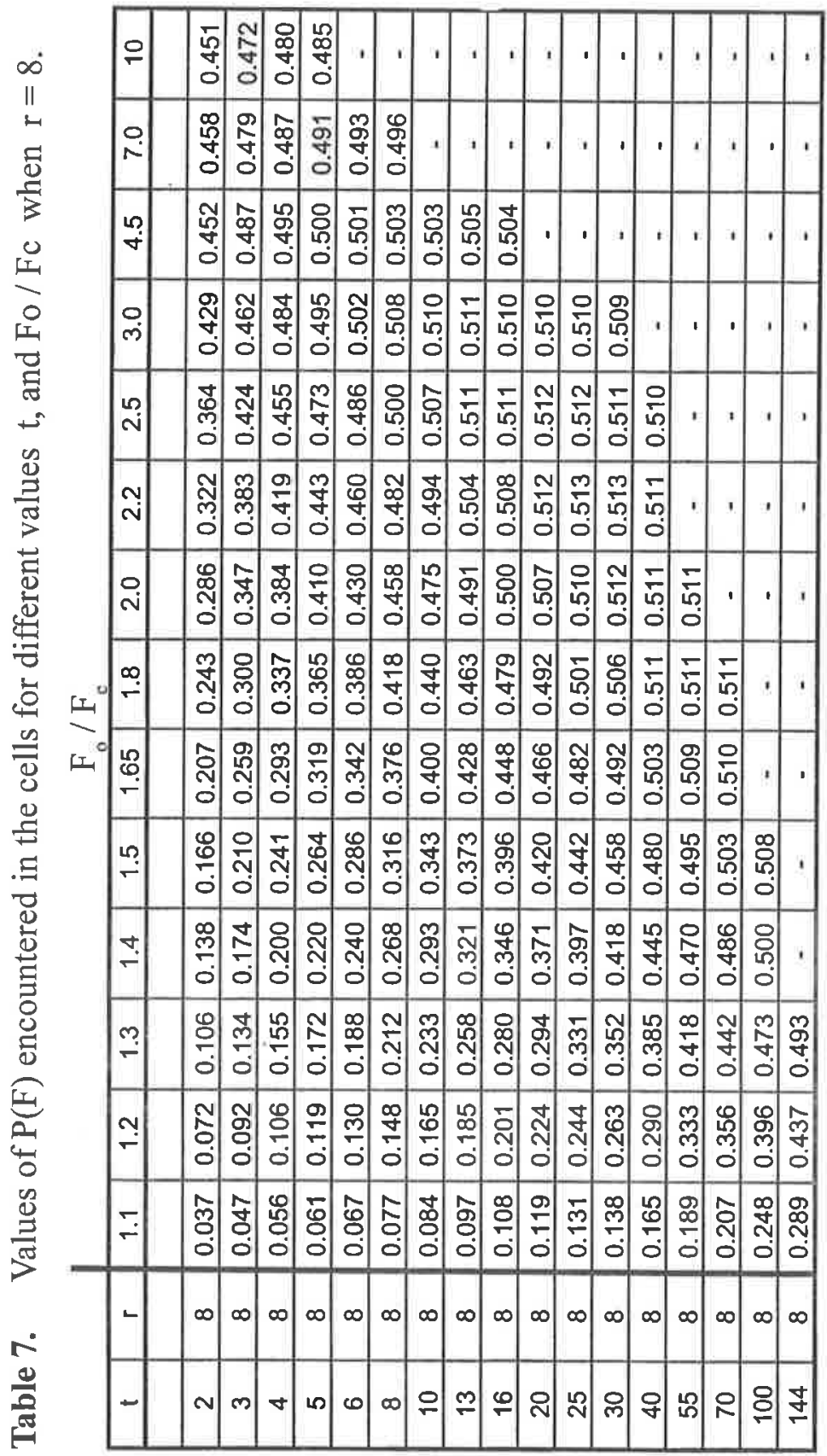




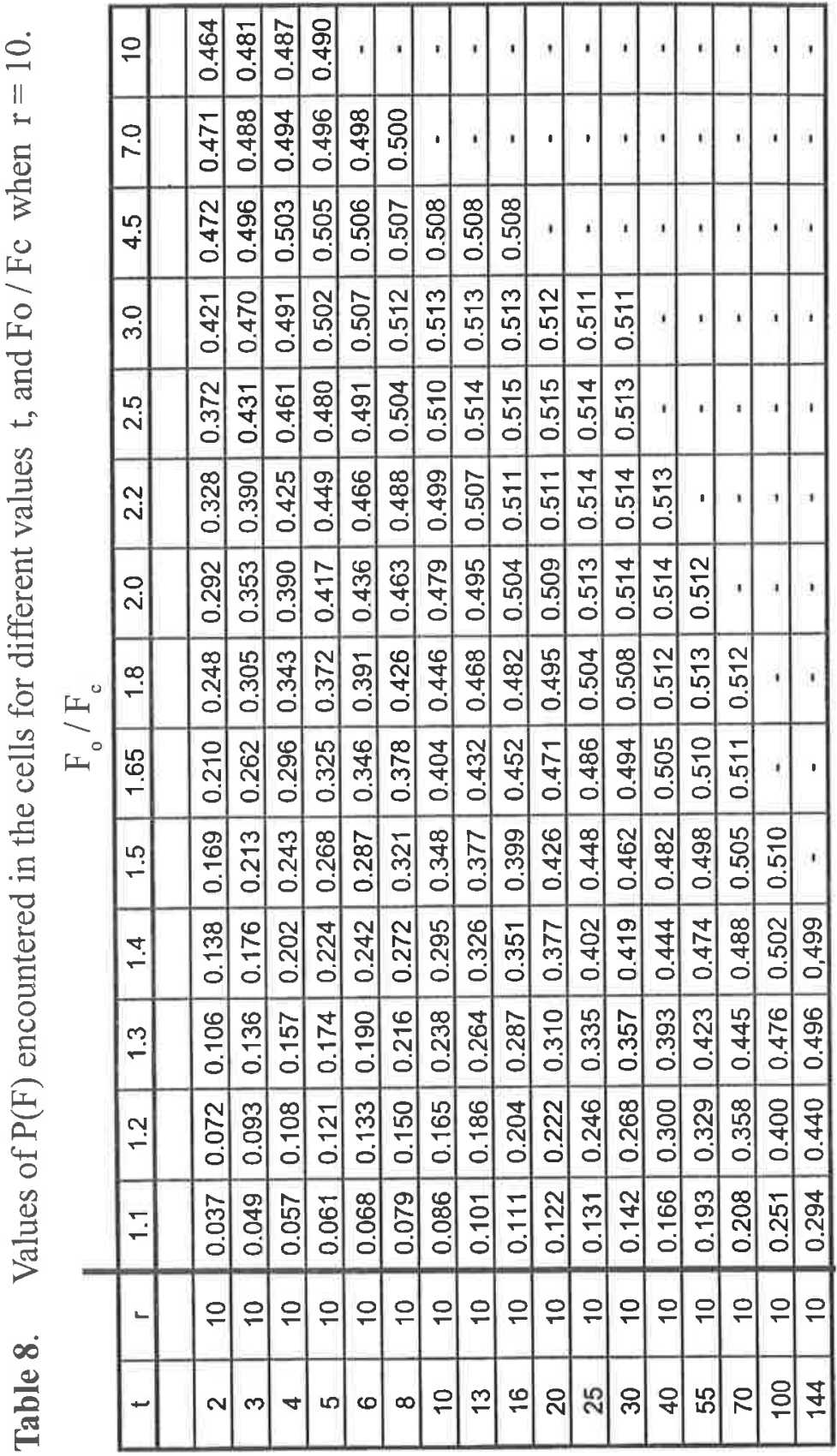




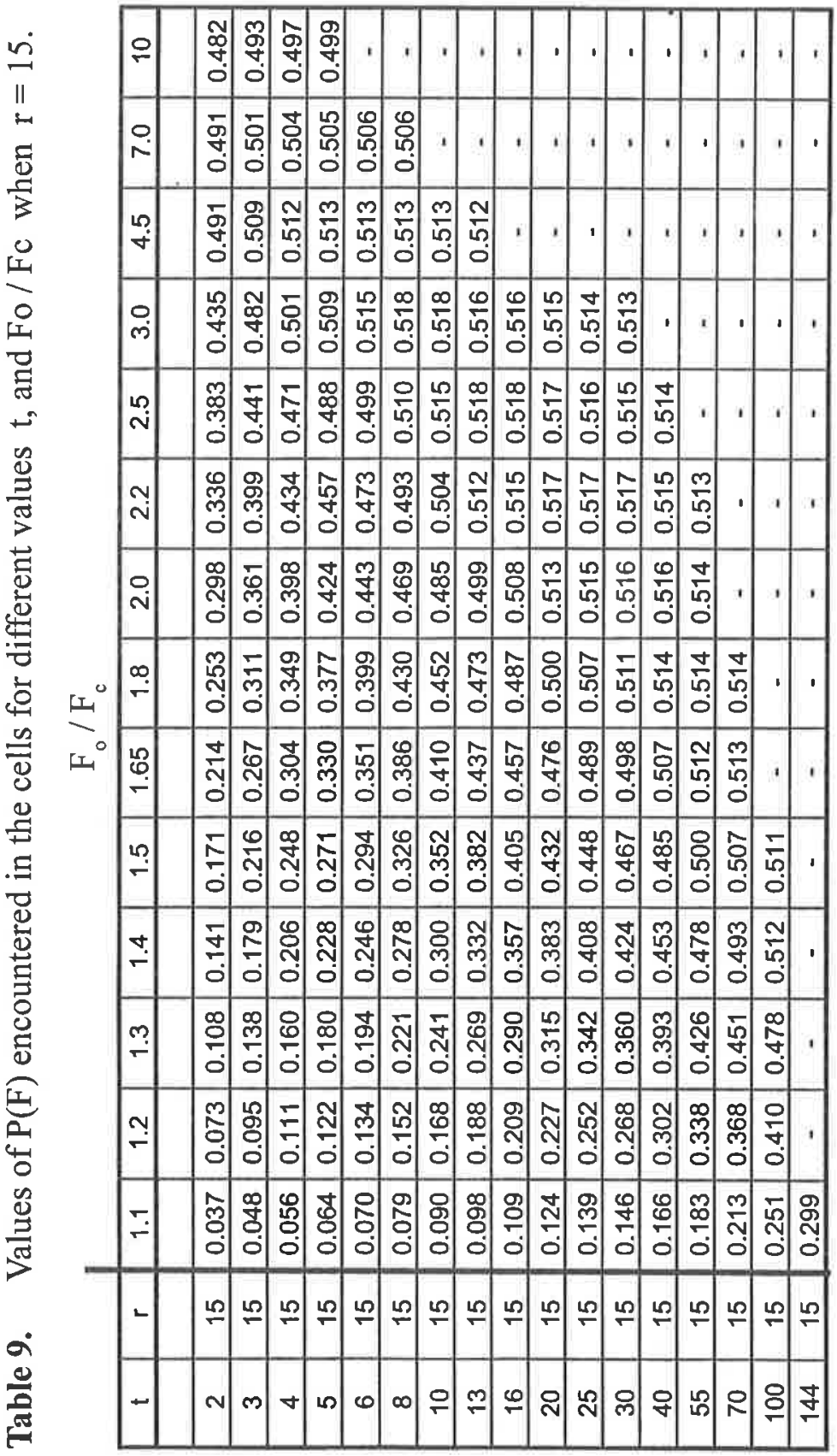




\section{REFERENCES}

CONAGIN, A.,1998. Discriminative Power of a Modified Bonferroni Test. Rev. Agricultura, 73: $31-46$.

CONAGIN, A., 1999. Discriminative Power of the Modified Bonferroni's

Test under General and Partial Null Hypothesis. Rev. Agricultura, 74: $117-126$.

SAS, 1990. SAS User's Guide. SAS Institute, chapters 11, 12 and 24. WINNER,B.J.; D.R.BROWN d K.M. MICHELS, 1991. Statistical Principles in Experimental Design. Mc Graw-Hill. 1057 p. 